\title{
A Rare Variant in PGAP2 Causes Autosomal Recessive Hyperphosphatasia with Mental Retardation Syndrome, with a Mild Phenotype in Heterozygous Carriers
}

\author{
Yonatan Perez, ${ }^{1}$ Ohad Wormser, ${ }^{1}$ Yair Sadaka, ${ }^{2}$ Ruth Birk, ${ }^{3}$ \\ Ginat Narkis, ${ }^{4}$ and Ohad S. Birk ${ }^{1,4}$ \\ ${ }^{1}$ The Morris Kahn Laboratory of Human Genetics, National Institute for Biotechnology in the Negev and \\ Faculty of Health Sciences, Ben Gurion University of the Negev, 84105 Beer Sheva, Israel \\ ${ }^{2}$ Pediatric Neurology Unit, Division of Pediatrics, Soroka University Medical Center, Faculty of Health Sciences, \\ Ben Gurion University of the Negev, 84101 Beer Sheva, Israel \\ ${ }^{3}$ Faculty of Health Sciences, Ariel University, Ariel, Israel \\ ${ }^{4}$ Genetics Institute, Soroka University Medical Center, Ben Gurion University of the Negev, 84101 Beer Sheva, Israel \\ Correspondence should be addressed to Ohad S. Birk; obirk@bgu.ac.il
}

Yonatan Perez and Ohad Wormser contributed equally to this work.

Received 31 March 2017; Revised 6 June 2017; Accepted 22 June 2017; Published 8 October 2017

Academic Editor: Paul Harrison

Copyright (c) 2017 Yonatan Perez et al. This is an open access article distributed under the Creative Commons Attribution License, which permits unrestricted use, distribution, and reproduction in any medium, provided the original work is properly cited.

\begin{abstract}
Mutations in genes involved in the biosynthesis of the glycosylphosphatidylinositol (GPI) anchor cause autosomal recessive glycosylation defects, with a wide phenotypic spectrum of intellectual disability, seizures, minor facial dysmorphism, hypotonia, and elevated serum alkaline phosphatase. We now describe consanguineous Bedouin kindred presenting with an autosomal recessive syndrome of intellectual disability and elevated serum alkaline phosphatase. Genome-wide linkage analysis identified 6 possible disease-associated loci. Whole-exome sequencing followed by Sanger sequencing validation identified a single variant in PGAP2 as the disease-causing mutation (C.554G $>$ A; p.185(R>Q)), segregating as expected within the kindred and not found in 150 Bedouin controls. The mutation replaces a highly conserved arginine residue with glutamine within the Fragl (FGF receptor activating) domain of PGAP2. Interestingly, this mutation is a known dbSNP variant (rs745521288, build 147) with a very low allele frequency (0.00000824 in dbSNP, no homozygotes reported), highlighting the fact that dbSNP variants should not be automatically ruled out as disease-causing mutations. We further showed that PGAP2 is ubiquitously expressed, but in line with the disease phenotype, it is highly transcribed in human brain, skeletal muscle, and liver. Interestingly, a mild phenotype of slightly elevated serum levels of alkaline phosphatase and significant learning disabilities was observed in heterozygous carriers.
\end{abstract}

\section{Introduction}

Biosynthesis of the glycosylphosphatidylinositol (GPI) anchor is a highly conserved process in eukaryotes. GPI-anchored proteins play an important role in membrane protein trafficking, cell-surface adhesion, and protection. Generation of GPI anchors results from a posttranslational modification of specific proteins, which is initiated by its synthesis at the endoplasmic reticulum. After synthesis, the GPI-anchor is continuously remodeled and transported to the cell membrane through the Golgi apparatus [1]. To date, mutations in six genes (PIGV, PIGY, PIGO, PGAP2, PIGW, and PGAP3) have been shown to cause hyperphosphatasia with mental retardation syndrome (HPMRS) in an autosomal recessive mode of inheritance (no autosomal dominant mutations have been reported thus far). Moreover, all of these genes are involved in GPI-anchor biosynthesis. PGAP2 is a noncatalytic protein which assists in attaching a saturated fatty acid to the anchor. This function of PGAP2 occurs in the Golgi apparatus and is required for stable association between GPI-anchored proteins and the cell-surface membrane rafts [2]. Seven different mutations in PGAP2 have been described to cause 


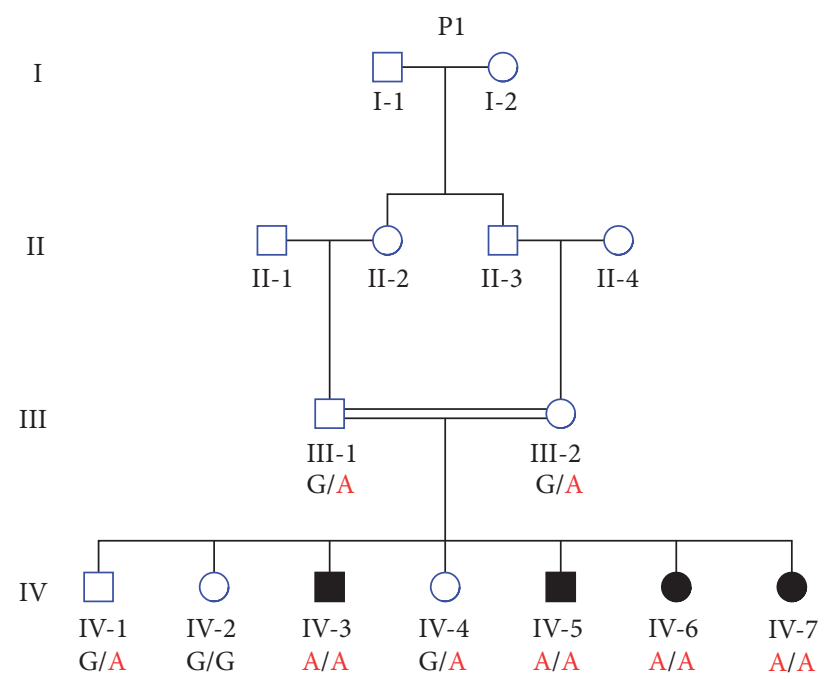

(a)

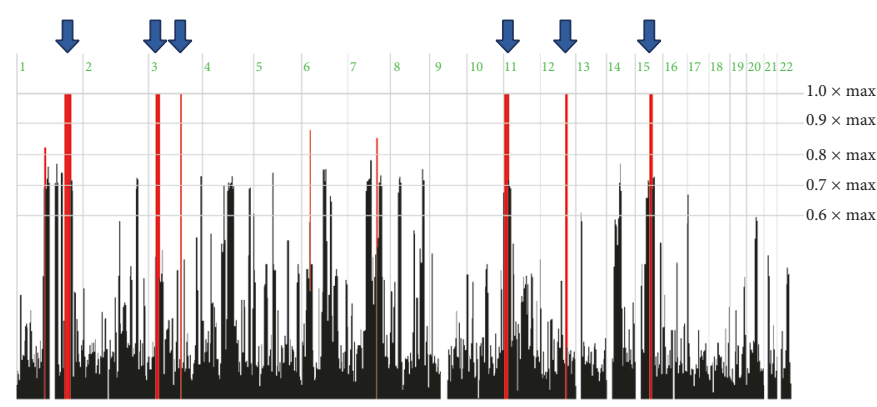

(c)

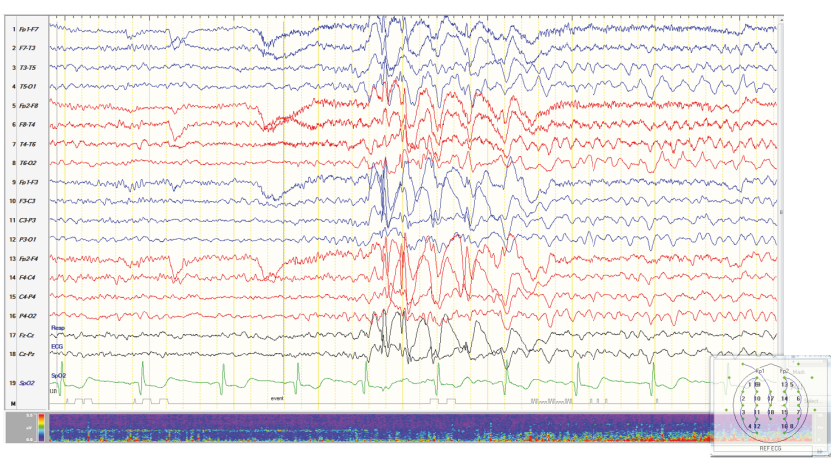

(b)

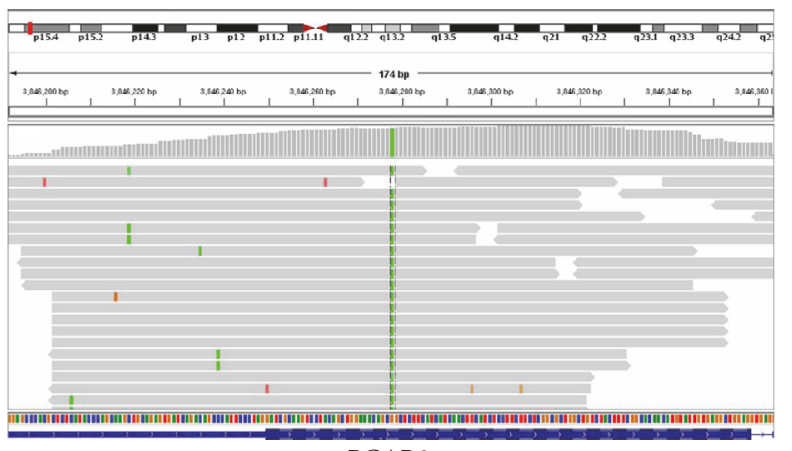

PGAP2

(d)

FIGURE 1: Pedigree of studied kindred, EEG recordings, homozygosity mapping, and the PGAP2 variant: (a) pedigree of consanguineous Bedouin kindred studied. Below each individual are the alleles of the PGAP2 mutation, whereas $\mathrm{G}$ (in black) represents the wild-type allele while A (in red) represents the mutant allele. (b) EEG recordings of patient IV-5 during wakefulness showing generalized interictal spike and slow epileptiform discharges more prominent anteriorly. (c) Homozygosity-Mapper plot; blue arrows present homozygous loci shared by three affected individuals. (d) Integrative Genomic Viewer (IGV) showing the PGAP2 variant.

hyperphosphatasia with mental retardation syndrome thus far [1-4]. In this study, we set out to decipher the molecular basis of apparently autosomal recessive hyperphosphatasia with mental retardation syndrome (HPMRS) in four individuals of a consanguineous Bedouin family (Figure 1(a)).

\section{Materials and Methods}

2.1. Subjects and Clinical Phenotyping. Nine family members of consanguineous Bedouin kindred were studied (Figure 1(a)). Clinical phenotyping was determined by an experienced team of pediatric neurologists and geneticists for all affected individuals, their parents, and siblings. DNA samples were obtained following approval of the Soroka Medical Center Internal Review Board.

2.2. Homozygosity Mapping. Genome-wide homozygosity mapping of three affected individuals (IV-3, IV-6, and IV7) (Figure 1(a)) was performed using CytoScan HD Array, which includes over 2.6 million functional markers across the entire genome and is suitable for copy number variation (CNV) detection as well as for genome-wide linkage analysis (Affymetrix, Inc.). Homozygosity mapping analysis was carried out using the open online software: HomozygosityMapper (http://www.homozygositymapper.org/) [5]. All physical positions mentioned are according to the GRCh37/hg19 genome assembly.

2.3. Sequencing Analysis. Whole-exome sequencing was performed by Centogene Company (Rostock, Germany), with $70-100 \mathrm{x}$ average coverage ( $95 \%$ targeted bases covered $>10 x)$. Library preparation was done using Nextera ${ }^{\circledR}$ Rapid Capture Exome Kit and sequencing was performed with Hiseq 2000 sequencing system (Illumina, Inc.). Data were analyzed using QIAGEN's Ingenuity ${ }^{\circledR}$ Variant Analysis ${ }^{\mathrm{TM}}$ software (https://www.qiagen.com/ingenuity) from QIAGEN Redwood City as previously described [6]. Using their filtering cascade, we excluded variants that are observed with 
an allele frequency greater than or equal to $1.0 \%$ of the genomes in the 1000 genomes project, NHLBI ESP exomes (All), or the Allele Frequency Community. In addition, we excluded variants which appeared in a homozygous state in our in-house whole-exome sequencing database of 150 Bedouin control samples. Furthermore, we kept variants which are predicted to have a deleterious effect upon protein coding sequences (e.g., frameshift, in-frame indel, stop codon change, missense or predicted to disrupt splicing by MaxEnt Scan) and variants which were experimentally observed to be associated with a phenotype: pathogenic, possibly pathogenic, or disease-associated according to the Human Gene Mutation Database (HGMD). Following the above filtering, of the remaining variants we selected only homozygous variants which were located within any of the homozygous loci that were identified by genome-wide homozygosity mapping. Validation and segregation analysis of the PGAP2 mutation was done via Sanger sequencing using the following primers: forward $5^{\prime}$ - GCCCATTCCCTAGGATCGC $-3^{\prime}$; reverse $5^{\prime}$ - AACACAATGGCAGCCAGTCC $-3^{\prime}$ (225 bp amplicon). Annealing temperature used was $60^{\circ} \mathrm{C}$, the extension time was set for 30 seconds, and PCR repeats were set for 40 cycles.

2.4. Multiple Sequence Alignment. Eight representative PGAP2 orthologues were selected for multiple sequence alignment (MSA). All protein sequences were taken from the National Center for Biotechnology Information GenBank (http://www.ncbi.nlm.nih.gov). The RefSeq sequence accession numbers for $D$. rerio, $X$. tropicalis, $R$. norvegicus, $M$. musculus, P. troglodytes, B. taurus, $H$. sapiens, and C. lupus familiaris of PGAP2 orthologues used for the analysis are NP 001013562.1, NP_001106477.1, XP_006229899.1, XP_006507706.1, XP_001159411.2, NP_001092581.1, NP_001243169.1, and XP_ 005633631.1, respectively. Protein MSA was performed using Clustal Omega program (http://www.ebi.ac.uk/Tools/ $\mathrm{msa} /$ clustalo/) [7].

2.5. PGAP2 Expression Analysis. A panel of cDNA samples was prepared from total RNA derived of 21 normal human tissues (Clontech Laboratories, Inc.), using Verso cDNA kit (Thermo Scientific ${ }^{\mathrm{TM}}$ ). Two sets of PCR primers were designed to amplify cDNA rather than genomic DNA of human PGAP2 and of glyceraldehyde 3-phosphate dehydrogenase $(G A P D H)$ housekeeping gene as a control. Primers used for $P G A P 2$ amplification (237 bp amplicon) are as follows: forward $5^{\prime}$ - AAACAGCGGCTCTTCATCAT -3'; reverse $5^{\prime}$ - CAAGCAGGACTGAAGGGTTC $-3^{\prime}$; primers used for GAPDH (452 bp amplicon) are as follows: forward $5^{\prime}$ - ACCACAGTCCATGCCATCAC - $3^{\prime}$; reverse $5^{\prime}$ - TCCACCACCCTGTTGCTGT $-3^{\prime}$. Annealing temperature used was $58^{\circ} \mathrm{C}$, the extension time was set for 30 seconds, and the PCR reaction repeats were of 35 cycles for both reactions.

\section{Results}

3.1. Clinical Characterization. Four individuals of consanguineous Bedouin family (Figure 1(a)) presented with a syndrome of intellectual disability and elevated serum alkaline phosphatase (Table 1). All affected individuals were born at term with normal birth weights, following uneventful pregnancies. There were no facial dysmorphism or any further abnormalities on physical examination of all patients, including thorough neurological examination, ruling out cranial, motor, sensory, gait, and cerebellar function abnormalities. Brain magnetic resonance imaging (MRI) of patients demonstrated no specific abnormal findings. Electroencephalogram (EEG) recordings done for patient IV-5 during wakefulness showed generalized interictal spike and slow epileptiform discharges that were more prominent anteriorly (Figure 1(b)). Karyotype and chromosomal microarrays (CMA) as well as molecular testing for fragile $\mathrm{X}$ syndrome were normal as well (Table 1).

3.2. Genetic Analysis. Genome-wide homozygosity mapping analysis of samples derived from three affected individuals (IV-3, IV-6, and IV-7) identified 6 different disease-associated loci (Table 2 and Figure 1(c)). Of the six genes previously shown to cause hyperphosphatasia with mental retardation syndrome (HPMRS), only one lies within any of these homozygous loci: PGAP2, located at position chr11:3,819,049$3,847,601$, residing within the chromosome 11 locus (Table 2).

Whole-exome sequencing data of individuals IV-3 (Figure 1(a)) were filtered for normal variants as described in Materials and Methods. Following the above filtering, four different rare variants remained: a c.3980A $>\mathrm{G}$, p.(N1327S) in $R F X$, a c.277A $>$ G, p.(I93V) in OR10A5, a c.1133G $>A$, p.(R323Q) in TUB, and a c.554G $>A$, p.(Arg185Gln) in $P G A P 2$, a gene previously associated with the disease phenotype (Figure $1(\mathrm{~d})$ ). These four potentially pathogenic variants which remained after the filtration cascade and which were located within the homozygous loci identified by genomewide homozygosity mapping were further assayed through segregation analysis (Table 3 ).

Only a single homozygous variant was found to reside within one of the above loci and to fully segregate as expected in autosomal recessive heredity (via Sanger sequencing) within all family members (i.e., parents were obligatory carriers, unaffected siblings were either carriers or homozygous for the wild-type allele, and affected individuals were all homozygous for the mutated allele; data not shown).

The mutation found is c.554G $>$ A, p.(Arg185Gln) in PGAP2, within the chromosome 11 locus (Figures 1(a) and 2(a)). The mutation resides within the Frag1 domain of the mature PGAP2 encoded protein (Figure 2(b)) and is predicted to replace a highly conserved arginine residue with a glutamine residue (Figure 2(c)). The mutation was also found to be possibly damaging by Polyphen- 2 predictions with a score of 0.942 (http://genetics.bwh.harvard.edu/pph2/). The $P G A P 2$ variant has been previously reported in the dbSNP database (https://www.ncbi.nlm.nih.gov/projects/SNP/) and in the Exome Aggregation Consortium (ExAC) Browser (http://exac.broadinstitute.org/) with an allele frequency of 0.000008122 (two carriers out of 246258 individuals; no homozygotes reported). In fact, there are no PGAP2 homozygous loss-of-function (LoF) mutations (stop gain, frameshift, or essential splice site mutations) or missense mutations which are reported in the Exome Aggregation Consortium 
TABle 1: Patients' clinical data.

\begin{tabular}{|c|c|c|c|c|}
\hline Patient & $\mathrm{Hx}$ & Physical exam & Lab measurements & Imaging + EEG \\
\hline $\begin{array}{l}\text { Patient } 1 \\
25 \text {-year-old male } \\
\text { sibling } \\
\text { IV-3 }\end{array}$ & $\begin{array}{l}\text { Mild mental retardation } \\
\text { Mood problems, } \\
\text { depression } \\
\text { Speech difficulties } \\
\text { Past history of febrile } \\
\text { convulsions }\end{array}$ & $\begin{array}{l}\text { No organ anomaly } \\
\text { No signs of dysmorphism } \\
\text { Normal cranial, motor, } \\
\text { sensory, gait, and } \\
\text { cerebellar exam }\end{array}$ & $\begin{array}{c}\text { Elevated alkaline } \\
\text { phosphatase: } \\
499 \text { IU/L at } 23 \text { yrs of age }\end{array}$ & $\begin{array}{l}\text { Normal brain CTA } \\
\text { Normal EEG }\end{array}$ \\
\hline $\begin{array}{l}\text { Patient } 2 \\
\text { 18-year-old male sibling } \\
\text { IV-5 }\end{array}$ & $\begin{array}{l}\text { Developmental delay } \\
\text { Moderate mental } \\
\text { retardation } \\
\text { Behavioral problems- } \\
\text { aggression } \\
\text { Past history of seizures } \\
\text { without fever }\end{array}$ & $\begin{array}{l}\text { No organ anomaly } \\
\text { No signs of dysmorphism } \\
\text { Normal cranial, motor, } \\
\text { sensory, gait, and } \\
\text { cerebellar exam }\end{array}$ & $\begin{array}{c}\text { Elevated alkaline } \\
\text { phosphatase: } \\
673 \mathrm{IU} / \mathrm{L} \text { at } 17 \mathrm{yrs} \text { of age }\end{array}$ & $\begin{array}{c}\text { Normal brain MRI } \\
\text { EEG: normal background } \\
\text { activity } \\
\text { Generalized spike and } \\
\text { wave interictal } \\
\text { epileptiform discharges }\end{array}$ \\
\hline $\begin{array}{l}\text { Patient } 3 \\
\text { 15-year-old female } \\
\text { sibling } \\
\text { IV-6 }\end{array}$ & $\begin{array}{l}\text { Mild mental retardation } \\
\text { Mood problems- } \\
\text { depression } \\
\text { Speech difficulties }\end{array}$ & $\begin{array}{c}\text { No organ anomaly } \\
\text { No signs of dysmorphism }\end{array}$ & $\begin{array}{c}\text { Elevated alkaline } \\
\text { phosphatase: } \\
>1000 \text { IU/L at } 10 \text { yrs of age }\end{array}$ & \\
\hline $\begin{array}{l}\text { Patient } 4 \\
\text { 11-year-old female } \\
\text { sibling } \\
\text { IV-7 }\end{array}$ & $\begin{array}{l}\text { Developmental delay } \\
\text { Mild mental retardation } \\
\text { Speech difficulties } \\
\text { Enuresis }\end{array}$ & $\begin{array}{c}\text { No organ anomaly } \\
\text { No signs of dysmorphism } \\
\text { Normal cranial, motor, } \\
\text { sensory, gait, and } \\
\text { cerebellar exam }\end{array}$ & $\begin{array}{c}\text { Elevated alkaline } \\
\text { phosphatase: } \\
1318 \text { IU/L at } 10 \text { yrs of age }\end{array}$ & Normal spinal MRI \\
\hline $\begin{array}{l}\text { Carrier } 1 \\
\text { 22-year-old male } \\
\text { sibling } \\
\text { IV-1 }\end{array}$ & $\begin{array}{c}\text { Reported Learning } \\
\text { disabilities without mental } \\
\text { retardation }\end{array}$ & $\begin{array}{c}\text { No organ anomaly } \\
\text { No signs of dysmorphism } \\
\text { Normal cranial, motor, } \\
\text { sensory, gait, and } \\
\text { cerebellar exam }\end{array}$ & $\begin{array}{l}\text { Mildly elevated alkaline } \\
\text { phosphatase: } \\
122 \mathrm{IU} / \mathrm{L} \text { at } 20 \mathrm{yrs} \text { of age }\end{array}$ & Normal brain CT scan \\
\hline $\begin{array}{l}\text { Carrier } 2 \\
\text { 19-year-old female } \\
\text { sibling } \\
\text { IV-4 }\end{array}$ & $\begin{array}{c}\text { Reported learning } \\
\text { disabilities without mental } \\
\text { retardation }\end{array}$ & $\begin{array}{l}\text { No organ anomaly } \\
\text { No signs of dysmorphism } \\
\text { Normal cranial, motor, } \\
\text { sensory, gait, and } \\
\text { cerebellar exam }\end{array}$ & $\begin{array}{l}\text { Mildly elevated alkaline } \\
\text { phosphatase: } \\
134 \text { IU/L at } 18 \text { yrs of age }\end{array}$ & - \\
\hline $\begin{array}{l}\text { Homozygous } \\
\text { wild-type } \\
\text { 24-year-old female } \\
\text { sibling } \\
\text { IV-2 }\end{array}$ & $\begin{array}{l}\text { High academic } \\
\text { performance }\end{array}$ & $\begin{array}{l}\text { No organ anomaly } \\
\text { No signs of dysmorphism } \\
\text { Normal cranial, motor, } \\
\text { sensory, gait, and } \\
\text { cerebellar exam }\end{array}$ & $\begin{array}{l}\text { Normal alkaline } \\
\text { phosphatase: } \\
84 \text { IU/L at } 23 \text { yrs of age }\end{array}$ & Normal brain CT \\
\hline
\end{tabular}

Patients IDs correspond with pedigree in Figure $1(\mathrm{a}) . \mathrm{Hx}=$ medical history; IU/L = international units per liter.

TABLE 2: Loci identified by homozygosity mapping.

\begin{tabular}{lccc}
\hline Chromosome & SNPs defining the borders of loci & Genomic positions (GRCh37/hg19) & Loci length (Mb) \\
\hline 1 & rs7534216-rs2070257 & $179627385-202117002$ & $\sim 2.5$ \\
3 & rs7644408-rs2276868 & $29891885-40498845$ & $\sim 10.6$ \\
3 & rs9876197-rs13094803 & $119099303-123565063$ & $\sim 4.5$ \\
11 & rs3852527-rs16906385 & $2826603-20552960$ & $\sim 17.8$ \\
12 & rs4762315-rs35735 & $97055302-100950371$ & $\sim 3.1$ \\
15 & rs2251480-rs1126308 & $54167341-63429086$ & $\sim 9.2$ \\
\hline
\end{tabular}

TABLE 3: Candidate variants within homozygous loci.

\begin{tabular}{lcccc}
\hline Gene & Genomic position & Transcript variant & Protein variant & dbSNP ID \\
\hline RFX & Chr15:56,382,731-56,535,483 & c.3980A $>$ G & p.(N1327S) & - \\
OR10A5 & Chr11:6,866,914-6,867,867 & c.277A $>$ G & p.(I93V) & rs540522650 \\
TUB & Chr11:8,060,180-8,127,654 & c.1133G $>$ A & p.(R323Q) & rs7498889465 \\
PGAP2 & Chr11:3,819,049-3,847,601 & c.554G $>$ A & p.(R185Q) & rs745521288 \\
\hline
\end{tabular}




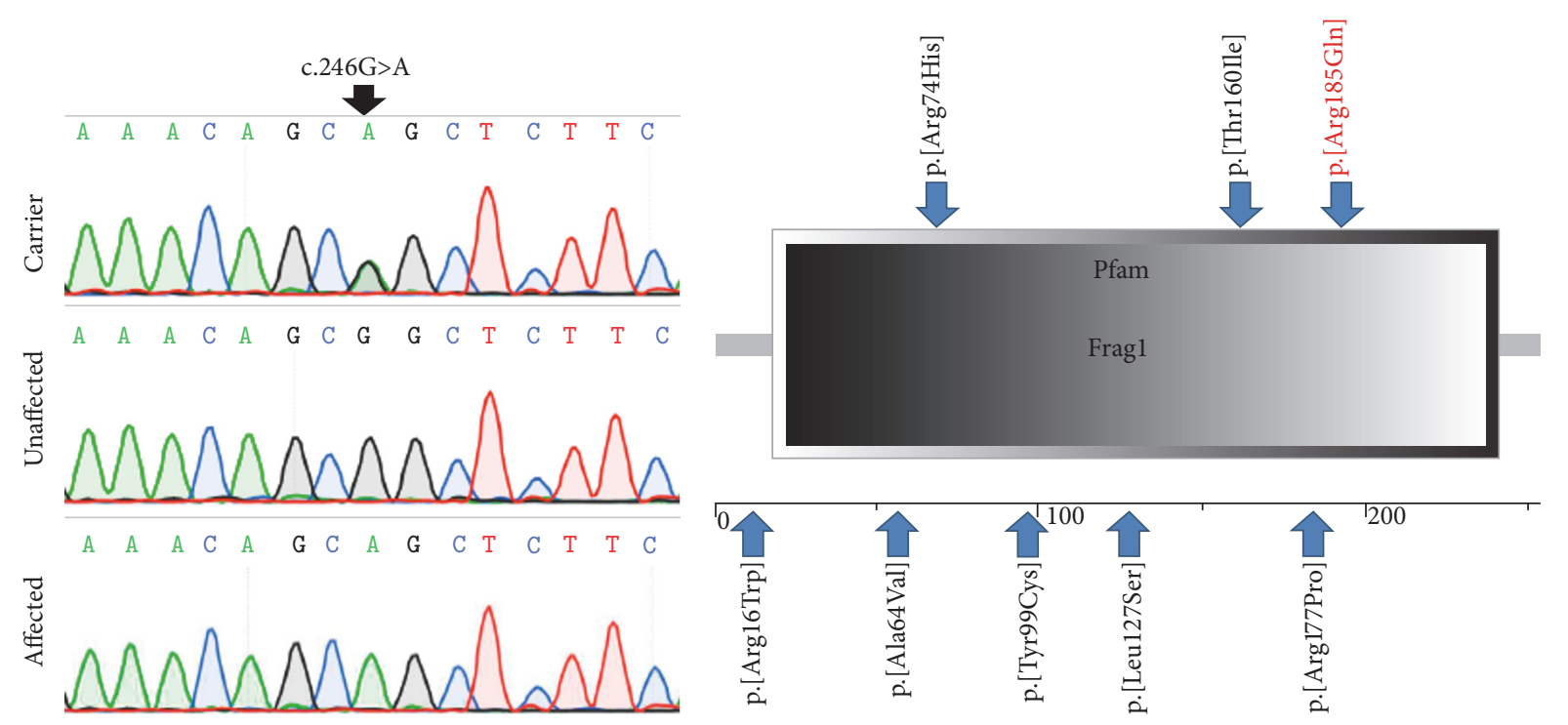

(a)

(b)

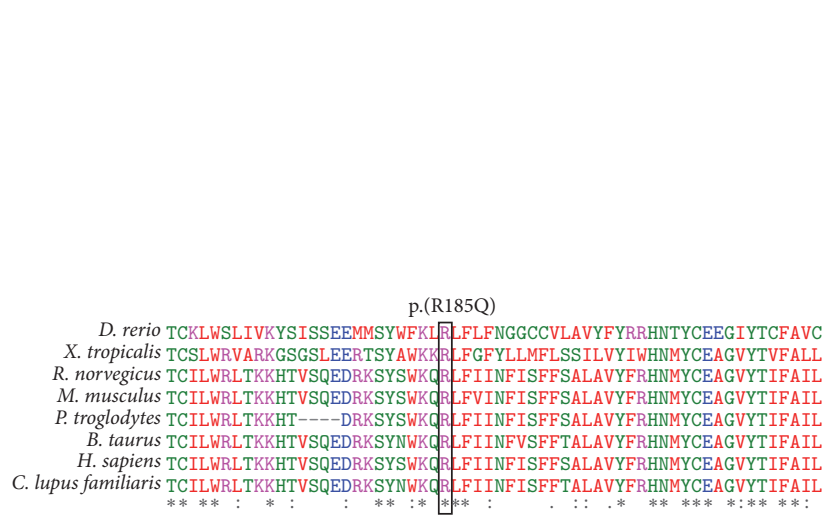

(c)

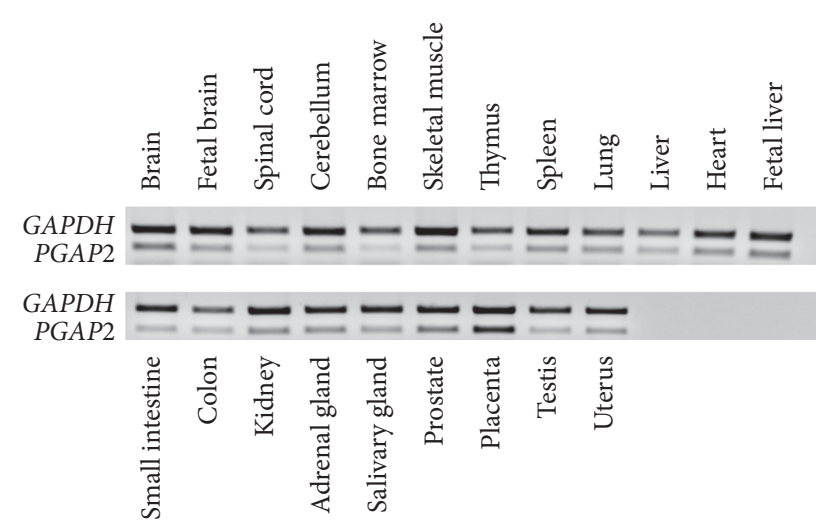

(d)

FIGURE 2: The PGAP2 mutation, domain architecture, conservation, and expression pattern: (a) Sanger sequencing of an obligatory carrier (III-1), an affected individual (IV-3), and an unaffected individual (IV-2). (b) The predicted domain architecture of PGAP2, showing its Fragl domain. The p.(Arg185Gln) mutation is marked in red. Blue arrows highlight additional PGAP2 mutations previously shown to cause hyperphosphatasia with mental retardation syndrome (HPMRS). (c) Multiple sequence alignment of selected PGAP2 orthologues. The mutation is predicted to cause p.(Arg185Gln) (boxed in black) at a highly conserved arginine residue within the putative Fragl domain of PGAP2. (d) RT-PCR of 21 normal human tissues demonstrating the expression pattern of PGAP2.

(ExAC). The PGAP2 transcript has multiple splicing isoforms and is highly conserved throughout evolution. Screening of the mutation in 150 ethnically matched controls (300 chromosomes) identified no carriers and no homozygous mutants (data not shown).

3.3. PGAP2 Expression Patterns. Analysis of PGAP2 expression in various normal human tissues by RT-PCR demonstrated that it is ubiquitous. However, while PGAP2 is moderately expressed in most tissues examined, it is highly transcribed in brain, cerebellum, skeletal muscle, heart, fetal liver, and placenta (Figure 2(d)).

\section{Discussion}

The kindred studied here, as is most of the Bedouin community in southern Israel, originates from Saudi Arabia. This community is unique in its high prevalence of consanguineous marriages, high birth rate, and extreme inbreeding within clans, leading to high incidence of monogenic diseases [8]. Here we describe an autosomal recessive form of hyperphosphatasia with mental retardation syndrome (HPMRS). Homozygosity mapping analysis of samples derived from three affected individuals identified 6 different disease-associated loci (Figure 1(c)). Amongst the known genes associated with HPMRS, PGAP2 was the only gene residing within any of the disease-associated loci identified by homozygosity mapping analysis. Exome sequencing combined with Sanger sequencing validation and segregation analysis identified a PGAP2 mutation as the only probable cause for this syndrome: c.554G $>$ A (NM_001256240.1), p.(Arg185Gln) (Figures 1(d) and 2(a)). The mutation segregated within the kindred as expected for autosomal recessive heredity (Figure 1(a)) and was not found in a homozygous or heterozygous state in 150 ethnically 
matched controls (data not shown). Clustal Omega analysis (http://www.ebi.ac.uk/Tools/msa/clustalo/) [7] showed that the p.(Arg185Gln) mutation in PGAP2 replaces a highly conserved arginine residue with glutamine within Frag1 domain (Figures 2(b) and 2(c)). Six different mutations within the Fragl domain have been previously described [14] (Figure 2(b)). All patients with mutations in this domain suffer from psychomotor retardation, intellectual disability, hypotonia, and increased serum alkaline phosphatase. While most features of this syndrome are shared between individuals with mutations in the PGAP2 Fragl domain, some phenotypic variability exists, with various degrees of microcephaly, seizures, and mild dysmorphic features. All our patients, homozygous for the same mutation, have in common all the basic features of the phenotype, yet have variable penetrance of seizures with no microcephaly or dysmorphism.

PGAP2 has multiple coding and noncoding isoforms. All coding transcripts harbor the c.554G $>$ A mutation. We demonstrated that $P G A P 2$ is highly transcribed in the human brain, skeletal muscle, heart, and fetal liver (Figure 2(d)) in line with the disease phenotype. PGAP2 is a noncatalytic highly conserved protein which assists in attaching a saturated fatty acid in the biosynthesis of glycosylphosphatidylinositol (GPI) anchor. Mutations in six different genes (PIGV, PIGY, PIGO, PGAP2, PIGW, and PGAP3) involved in GPIanchor biosynthesis have been shown to cause hyperphosphatasia with mental retardation syndrome (HPMRS). All HPMRS cases described to date, regardless of the specific mutated gene, are inherited in an autosomal recessive fashion. To date, 7 mutations in PGAP2 gene have been identified as a cause of hyperphosphatasia with mental retardation syndrome (HPMRS) (Figure 2(b)).

Interestingly, we show that two carriers (IV-1 and IV-4) also presented slightly high levels of alkaline phosphatase in serum and have also been reported to have significant learning disabilities, though without mental retardation (Table 1). In contrast, their sibling (IV-2) which is homozygous for the wild-type allele reported to have high academic performances (having completed a university Master's degree). It would be of interest to examine whether similar cases of a mild phenotype have been reported for other heterozygous individuals, carrying different mutations in PGAP2.

Altogether, our data demonstrate that the autosomal recessive syndrome of hyperphosphatasia with mental retardation in this family is caused by a rare homozygous variant in PGAP2 which was previously reported in dbSNP database and that a mild phenotype could be observed in heterozygous carriers.

\section{Abbreviations}

MRI: Magnetic resonance imaging

CT: $\quad$ Computed tomography

CTA: Computed tomography angiography

RT-PCR: Reverse transcription polymerase chain reaction

Mb: $\quad$ Million bases

ESP: $\quad$ Exome Sequencing Project

NHLBI: National Heart, Lung, and Blood Institute
NCBI: National Center for Biotechnology Information

WT: Wild-type

IRB: Institutional review board

MSA: Multiple sequence alignment

HGMD: Human gene mutation database

SNP: Single nucleotide polymorphism

ExAC: Exome Aggregation Consortium

IGV: Integrative Genomic Viewer

HPMRS: Hyperphosphatasia with mental retardation syndrome

ALP: Alkaline phosphatase.

\section{Additional Points}

Web Resources. The following resources were used: UCSC genome browser: https://genome.ucsc.edu/; Chromas: http:// technelysium.com.au/; HaploPainter: http://haplopainter .sourceforge.net/index.html; Primer3 (V.0.4.0): http://frodo .wi.mit.edu/primer3/; SNP database: https://www.ncbi.nlm .nih.gov/projects/SNP/; Clustal Omega software: http://www .ebi.ac.uk/Tools/msa/clustalo/; NHLBI Exome Sequencing Project, Seattle, WA: http://evs.gs.washington.edu/EVS/; Online Mendelian Inheritance in Man (OMIM): http://www .omim.org/; Biotechnology Information GenBank: https:// www.ncbi.nlm.nih.gov/; Homozygosity-Mapper: http:// www.homozygositymapper.org/; 1000 genomes project: http://www.1000genomes.org/; PolyPhen-2: http://genetics .bwh.harvard.edu/pph2/; ExAC Browse: http://exac.broadinstitute.org/.

\section{Conflicts of Interest}

The authors declare that there are no conflicts of interest regarding the publication of this paper.

\section{Authors' Contributions}

Yonatan Perez and Ohad Wormser equally contributed to this paper.

\section{Acknowledgments}

The authors thank Dr. Khalil Elbedour for recruiting the family to this study and thank the family members who agreed to participate. Funding for this research was provided by the Legacy Heritage Bio-Medical Program of the Israel Science Foundation (Grant no. 1798/16 awarded to Ohad S. Birk and Grant no. 1520/09 awarded to Ruth Birk and Ohad S. Birk).

\section{References}

[1] L. Hansen, H. Tawamie, Y. Murakami et al., "Hypomorphic mutations in PGAP2, encoding a GPI-anchor-remodeling protein, cause autosomal-recessive intellectual disability," American Journal of Human Genetics, vol. 92, no. 4, pp. 575-583, 2013. 
[2] P. M. Krawitz, Y. Murakami, A. Rieß et al., "PGAP2 mutations, affecting the GPI-anchor-synthesis pathway, cause hyperphosphatasia with mental retardation syndrome," American Journal of Human Genetics, vol. 92, no. 4, pp. 584-589, 2013.

[3] A. Jezela-Stanek, E. Ciara, D. Piekutowska-Abramczuk et al., "Congenital disorder of glycosylphosphatidylinositol (GPI)anchor biosynthesis-The phenotype of two patients with novel mutations in the PIGN and PGAP2 genes," European Journal of Paediatric Neurology, vol. 20, no. 3, pp. 462-473, 2016.

[4] M. I. Naseer, M. Rasool, M. M. Jan et al., "A novel mutation in PGAP2 gene causes developmental delay, intellectual disability, epilepsy and microcephaly in consanguineous Saudi family," Journal of the Neurological Sciences, vol. 371, pp. 121-125, 2016.

[5] D. Seelow, M. Schuelke, F. Hildebrandt, and P. Nürnberg, "HomozygosityMapper-An interactive approach to homozygosity mapping," Nucleic Acids Research, vol. 37, no. 2, pp. W593-W599, 2009.

[6] Y. Perez, Z. Shorer, K. Liani-Leibson et al., "SLC30A9 mutation affecting intracellular zinc homeostasis causes a novel cerebrorenal syndrome," Brain, vol. 140, no. 4, pp. 928-939, 2017.

[7] F. Sievers, A. Wilm, D. Dineen et al., "Fast, scalable generation of high-quality protein multiple sequence alignments using Clustal Omega," Molecular Systems Biology, vol. 7, article 539, 2011.

[8] B. Markus, I. Alshafee, and O. S. Birk, "Deciphering the finestructure of tribal admixture in the Bedouin population using genomic data," Heredity, vol. 112, no. 2, pp. 182-189, 2014. 

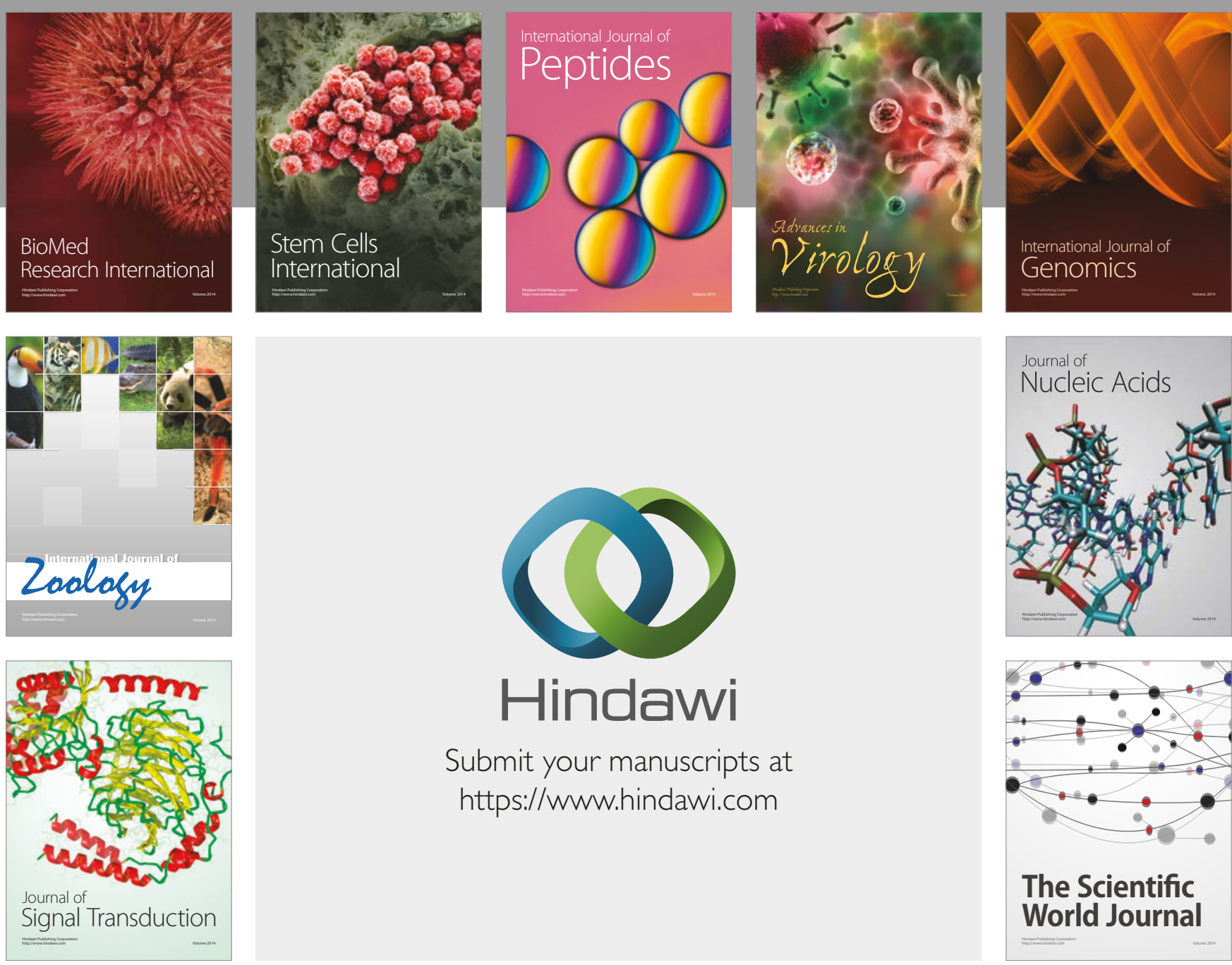

Submit your manuscripts at

https://www.hindawi.com
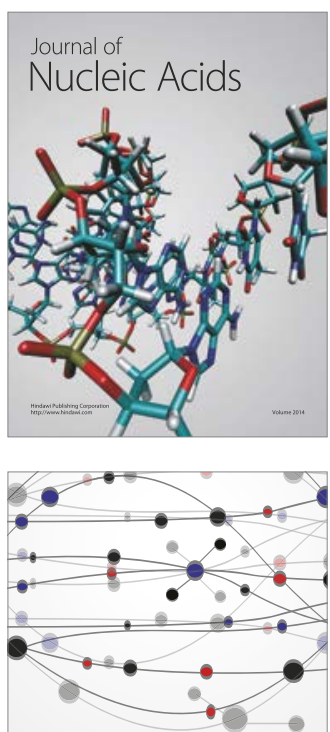

The Scientific World Journal

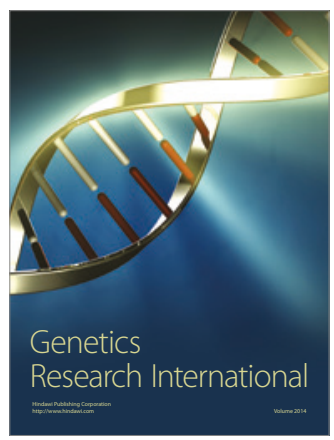

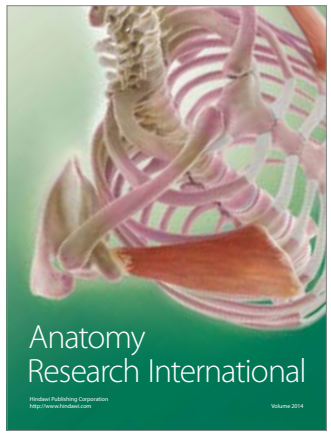

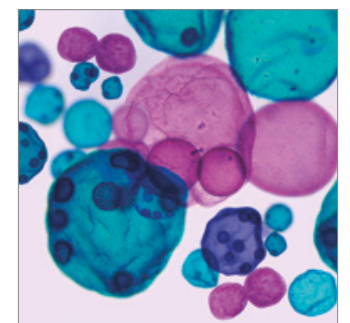

International Journal of Microbiology
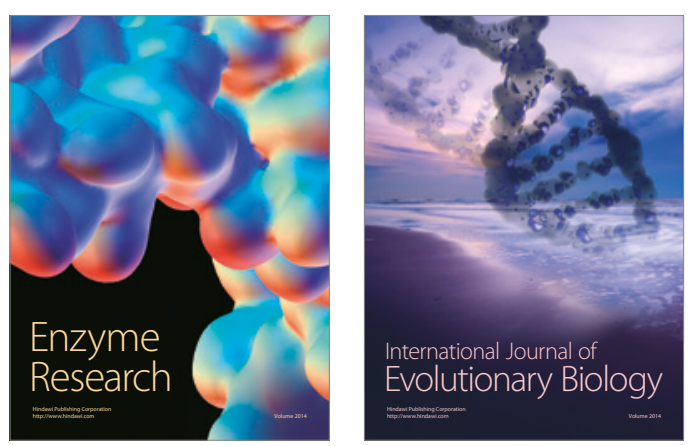
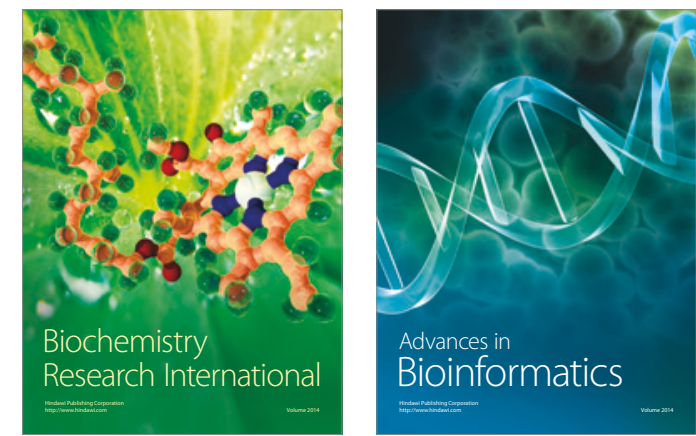

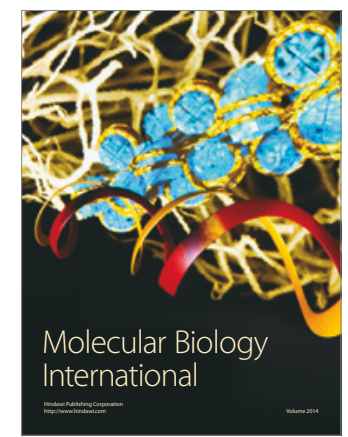

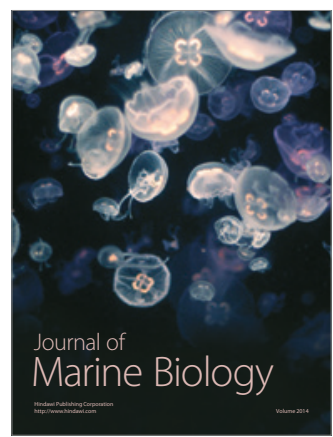

